ENFOQUES JURIDICOS

REVISTA MULTIDISCIPLINAR DEL CEDECS

ISSN 26832070

Número 04

julio-diciembre 2021
Artículo: "Los derechos de autor en el ámbito digital. Un necesario sistema de equilibrio de libertades en México"

José Joaquín Piña Mondragón

DOI: https://doi.org/10.25009/ej.v0i04.2570

\title{
Los derechos de autor en el ámbito digital. Un necesario sistema de equilibrio de libertades en México
}

Recibido 02 marzo 2021-Aceptado 26 abril 2021.

José Joaquín Piña Mondragón*

Centro de Ingeniería y Desarrollo Industrial-CONACyT

jpina@conacyt.mx

RESUMEN: El presente artículo analiza los principales instrumentos jurídicos que con ocasión de la entrada en vigor del Tratado Integral y Progresista de Asociación Transpacífico (TIPAT) y del Tratado entre México, Estados Unidos y Canadá (T-MEC), requirió la armonización de diversas disposiciones de la Ley Federal del Derecho de Autor (LFDA), con el propósito de adaptar las nuevas formas de almacenamiento, puesta a disposición y comunicación pública de obras protegidas por derechos de autor a través de canales digitales que han facilitado el acceso a los contenidos de manera abierta a través de internet y prácticamente sin restricciones, abriendo con ello una nueva era en materia de protección de los derechos de autor y el uso de obras en el entorno digital en México. Sin embargo, la regulación de
ABSTRACT: This article analyzes the main legal instruments that, on the occasion of the entry into force of the Comprehensive and Progressive Agreement for TransPacific Partnership (CPTPP) and United States, Mexico and Canada Agreement (UMSCA), required the harmonization of various provisions of the Federal Copyright Law (LFDA), with the purpose of adapting the new forms of storage, making available and public communication of works protected by copyright through digital channels that have facilitated access to the contents in a manner open through the internet and practically without restrictions, thus opening a new era in terms of copyright protection and the use of works in the digital environment in Mexico. However, the regulation of content through digital media implies important challenges

* Licenciado, Maestro y Doctor en Derecho por la Facultad de Derecho de la UNAM. Investigador Cátedra CONACyT en el Centro de Ingeniería y Desarrollo Industrial. Investigador Nacional nivel I del SNI.

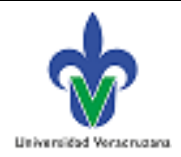


ENFOQUES JURIDICOS

REVISTA MULTIDISCIPLINAR DEL CEDECS

ISSN 26832070

Número 04

julio-diciembre 2021
Artículo: "Los derechos de autor en el ámbito digital. Un necesario sistema de equilibrio de libertades en México"

José Joaquín Piña Mondragón

DOI: https://doi.org/10.25009/ej.v0i04.2570 contenidos a través de medios digitales implica retos importantes para garantizar las libertades y derechos fundamentales de los usuarios y para proteger de forma efectiva los derechos de los autores respecto de sus obras que circulan en internet. De no existir el equilibrio necesario entre ambos derechos, los fundamentos de la sociedad de la información no se sostendrán, ni tampoco el desarrollo de las tecnologías de la información y knowledge society, TIC. comunicación (TIC).

Palabras clave: ámbito digital, derechos de autor, sociedad del conocimiento, TIC. to guarantee the fundamental freedoms and rights of users and to effectively protect the rights of authors with respect to their works that circulate on the internet. In the absence of the necessary balance between both rights, the basis of the information society will not be sustained, nor will the development of information and communication technologies (ICT).

Keywords: digital environment, copyright,

SUMARIO: Introducción. 1. Marco jurídico que regula las formas de uso de las obras y contenidos en el ámbito digital en México. 2. Derechos de autor y TIC. 3. La protección de los derechos de autor frente a los derechos y las libertades fundamentales de los usuarios en el ámbito digital. Conclusiones. Fuentes de Consulta.

\section{Introducción}

En el contexto de la actual sociedad de la información y del conocimiento en que vivimos un tema fundamental es la protección de los derechos de propiedad intelectual en el ámbito digital. Lo anterior ante la facilidad que representa para los usuarios de internet acceder a cualquier tipo de información y contenidos de manera abierta que, en muchas ocasiones, implica la vulneración de los derechos intelectuales de los autores respecto de sus obras y contenidos.

Ya los primeros gurús de internet predecían la creación de una comunidad global más abierta, en la que la información y el conocimiento se compartirían e intercambiarían en el ciberespacio a través de una red interconectada de millones de ordenadores y usuarios en el planeta.

Todos estos progresos auspiciados por la emergencia de las TIC nos acercan a ese objetivo en la sociedad del conocimiento, un paradigma de organización cultural, económico, industrial y social, caracterizado por la eliminación de las barreras naturales del tiempo y el espacio, que ha hecho posible el acercamiento de personas y sociedades anteriormente lejanas, a la información como su principal insumo.

Actualmente, se considera que una sociedad es más abierta, democrática y avanzada, en la medida que permite mayor difusión de la información y contenidos de manera

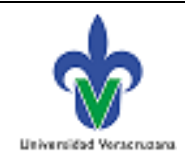

pp. $39-60$ 
ENFOQUES JURIDICOS

REVISTA MULTIDISCIPLINAR DEL CEDECS

ISSN 26832070

Número 04

julio-diciembre 2021
Artículo: "Los derechos de autor en el ámbito digital. Un necesario sistema de equilibrio de libertades en México"

José Joaquín Piña Mondragón

DOI: https://doi.org/10.25009/ej.v0i04.2570

instantánea. De esta forma, la información se constituye en la materia prima para el desarrollo de la sociedad del conocimiento.

En efecto, en la medida que se divulga más cantidad de contenidos e información, aumenta el interés de los usuarios de las redes digitales para acceder a ellos, al tiempo que se incrementan las herramientas tecnológicas para poner a disposición de manera gratuita e instantánea las obras. Esa es precisamente una de las principales razones del incremento en la demanda de redes de alta velocidad y dispositivos móviles, que permiten acceder en prácticamente cualquier lugar y momento a animaciones, videos, textos, recursos musicales, programas de cómputo, código fuente, íconos, gráficos y diversas obras digitales que se almacenan y comparten a través de internet.

Al respecto, surge la problemática respecto a ¿cómo garantizar el equilibrio y estabilidad necesarios entre la protección de los derechos de los autores con relación a sus obras que circulan en internet y los derechos y libertades fundamentales de los usuarios que acceden a ellas, como el derecho a la información, libertad de expresión y de internet?, ambos derechos reconocidos en acuerdos internacionales y legislaciones nacionales. Responder esta interrogante en el entorno digital se convierte en un tema complejo; máxime si tomamos en cuenta que ese sistema de equilibrio deriva de uno de los pilares fundamentales del derecho de autor, como es la difusión cultural mediante el acceso a las obras (Guerra y Hernández, 2021: 15).

De esta forma, la investigación consta de tres apartados, el primero, relativo al análisis del marco jurídico que regula las formas de uso de las obras y contenidos que circulan en el entorno digital, haciendo un recorrido por las principales regulaciones internacionales, como los Tratados de Internet de la OMPI, e iniciativas como SOPA, PIPA y ACTA, hasta llegar a las disposiciones contenidas en TIPAT y T-MEC, que fueron recogidas en un proceso de armonización legislativa con la reciente reforma a la LFDA en México, publicada en el Diario Oficial de la Federación de fecha 1 de julio de 2020. Lo anterior nos permitirá en un segundo apartado, centrar el análisis en torno a la relación existente entre los derechos de autor y las TIC, a efecto de plantear, en el tercer apartado, la necesidad de una relación equilibrada entre la protección de los derechos de autor de los creadores frente a las libertades fundamentales de los usuarios de contenidos en el entorno digital, que giran en torno al derecho de acceso a la información, la educación, la libertad de expresión y de internet.

\section{Marco jurídico que regula las formas de uso de las obras y contenidos en el ámbito digital en México}

Con la evolución vertiginosa de los avances tecnológicos, surgieron los primeros tratados para regular las relaciones entre los autores y los usuarios de obras en el ámbito digital. Así, las primeras reformas legales tendentes a establecer soluciones ante los nuevos desafíos

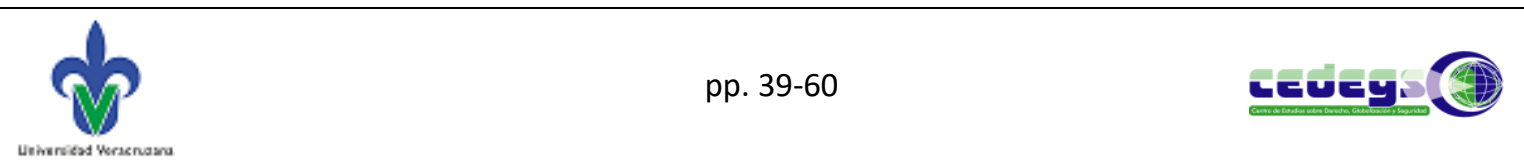


ENFOQUES JURIDICOS

REVISTA MULTIDISCIPLINAR DEL CEDEGS

ISSN 26832070

Número 04

julio-diciembre 2021
Artículo: "Los derechos de autor en el ámbito digital. Un necesario sistema de equilibrio de libertades en México"

José Joaquín Piña Mondragón

DOI: https://doi.org/10.25009/ej.v0i04.2570

que plantea la evolución de los avances tecnológicos frente a los derechos de autor se produjeron en el contexto internacional.

Al respecto, el Acuerdo sobre los Aspectos de los Derechos de Propiedad Intelectual relacionados con el Comercio (ADPIC), adoptado en Marrakesh el 15 de abril de 1994, en el marco de la Ronda Uruguay (1986-1994) por la que se crea la Organización Mundial de Comercio (OMC), incorporó como propios los principios fundamentales establecidos por el Convenio de París para la Protección de la Propiedad Industrial; del Convenio de Berna para la Protección de las Obras Literarias y Artísticas; y de la Convención de Roma sobre la protección de los artistas intérpretes o ejecutantes, los productores de fonogramas y los organismos de radiodifusión; a los cuales añadió el principio de nación más favorecida, característico del sistema multilateral de comercio.

Por otra parte, la Organización Mundial de la Propiedad Intelectual (OMPI), en respuesta a la primera oleada que trajo consigo internet y la emergencia de las tecnologías digitales, en diciembre de 1996 celebró una Conferencia Diplomática para diseñar el marco jurídico internacional que permitiera enfrentar los nuevos desafíos que las TIC planteaban respecto a la protección de los derechos intelectuales.

En el marco de dicha Conferencia, los Estados participantes suscribieron dos acuerdos internacionales: a) el Tratado de la OMPI sobre Derecho de Autor (TODA), que entró en vigor el 6 de marzo de 2002 y b) el Tratado de la OMPI sobre Interpretación o Ejecución de Fonogramas (TOIEF), que entró en vigor el 20 de marzo del mismo año y de los cuales México es Parte.

Ambos instrumentos denominados "Tratados de Internet", son resultado de la necesidad de adaptar y proteger los derechos de autor en el ámbito digital. Su trascendencia estriba en la actualización de la protección internacional de los derechos de autor y conexos con relación a la denominada "agenda digital". En particular, la diseminación de contenidos protegidos en redes digitales y la mejora de los medios para su protección a nivel mundial.

La aportación concebida en ambos acuerdos consistió en el establecimiento de un nuevo derecho exclusivo conocido como "puesta a disposición", que el artículo 10 del TOIEF refiere como

el derecho exclusivo de autorizar la puesta a disposición del público de sus interpretaciones o ejecuciones fijadas en fonogramas, ya sea por hilo o por medios inalámbricos de tal manera que los miembros del público puedan tener acceso a ellas desde el lugar y en el momento que cada uno de ellos elija.

Este derecho comprende, por un lado, la puesta a disposición a través de medios digitales $y$, por otro, el empleo de medios tecnológicos que permitan proteger los derechos de autor en el ámbito digital. Por su parte, el TODA dio libertad para que las legislaciones de cada Estado caracterizaran este nuevo derecho, ya sea como comunicación al público o

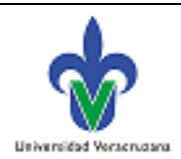

pp. $39-60$ 
ENFOQUES JURÍDICOS

REVISTA MULTIDISCIPLINAR DEL CEDECS

ISSN 26832070

Número 04

julio-diciembre 2021
Artículo: "Los derechos de autor en el ámbito digital. Un necesario sistema de equilibrio de libertades en México"

José Joaquín Piña Mondragón

DOI: https://doi.org/10.25009/ej.v0i04.2570

distribución, o incluso mediante una combinación de derechos prexistentes (OMPI, 2015: 2).

En concreto, los Tratados de Internet actualizaron los principios jurídicos para sustentar la protección internacional de los derechos autorales y conexos en el entorno digital; estableciendo los parámetros para la creación de un sistema más equilibrado de protección en rubros como la transmisión interactiva de contenidos, limitaciones al derecho de autor y promoción de tecnologías que faciliten la distribución y uso de contenidos creativos.

De esta forma, en el preámbulo de las Declaraciones concertadas relativas al TODA, la OMPI reconoció el impacto de las TIC en la producción y distribución de contenidos, introduciendo soluciones frente a los retos planteados a partir de la revolución tecnológica que trajo consigo el internet.

\section{a) Tratado de la OMPI sobre derecho de autor}

Este Acuerdo reformuló lo establecido por la OMPI en la revisión del Convenio de Berna del 24 julio de 1971, cuando la posibilidad de digitalización y el uso de las nuevas TIC se encontraban en una fase inicial.

La nota 1 del artículo 1 del Acuerdo, dispone que el derecho de reproducción y las excepciones al mismo, también son aplicables a la utilización de obras en el entorno digital, entendiendo que el almacenamiento en un soporte electrónico de obras protegidas implica un acto de reproducción en términos del artículo 9 del Convenio de Berna. En el mismo sentido, el artículo 4 asimila la protección de los programas de cómputo, cualquiera que sea su modo o forma de expresión, como obras literarias en términos del artículo 2 del Convenio de Berna.

Por su parte, el artículo 8 reconoce el derecho exclusivo de los autores de obras literarias y artísticas de autorizar cualquier comunicación pública de sus obras por medios alámbricos o inalámbricos, desde el lugar y en el momento que elijan. De lo anterior, podemos desprender que el almacenamiento en forma digital, aunque sea de carácter temporal, implica un acto de reproducción, en tanto que la puesta a disposición constituye en sí misma la comunicación pública de las obras.

El artículo 11 deja la posibilidad para que cada Parte, en el marco del Acuerdo, pueda adoptar las medidas de carácter tecnológico para restringir aquellos actos que no se encuentren autorizados por los autores o permitidos por la ley. Al respecto, el artículo 12 dispone que las Partes establecerán las medidas contra aquella persona que "suprima o altere sin autorización cualquier información electrónica sobre la gestión de derechos"; y respecto al que distribuya ejemplares de una información electrónica sobre gestión de derechos a sabiendas de que ésta ha sido suprimida o alterada sin autorización y la distribuya, emita o comunique al público.

Finalmente, el artículo 14 compromete a las Partes contratantes a disponer las medidas necesarias para asegurar la plena aplicación del Tratado y establecer los procedimientos

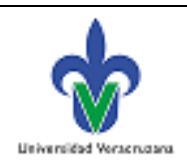

pp. $39-60$ 
ENFOQUES JURIDICOS

REVISTA MULTIDISCIPLINAR DEL CEDECS

ISSN 26832070

Número 04

julio-diciembre 2021
Artículo: "Los derechos de autor en el ámbito digital. Un necesario sistema de equilibrio de libertades en México"

José Joaquín Piña Mondragón

DOI: https://doi.org/10.25009/ej.v0i04.2570

para la observancia de los derechos, adoptando medidas eficaces para disuadir cualquier actividad que infrinja los mismos.

\section{b) Tratado de la OMPI sobre interpretación o ejecución de fonogramas}

Al igual que TODA, este Acuerdo destaca su independencia con relación a otros instrumentos multilaterales en materia de protección de la propiedad intelectual. Dicha situación atiende al interés de las Partes por puntualizar que una eventual inobservancia no daría lugar a sanciones por parte de la OMC (Rubí, 2007: 101).

Un aspecto a destacar de este tratado es el relativo al reconocimiento de los derechos patrimoniales de los productores de fonogramas para autorizar la reproducción, distribución, alquiler y puesta a disposición al público de sus fonogramas, que en términos de la Convención de Roma sobre la protección de los artistas intérpretes o ejecutantes, los productores de fonogramas y los organismos de radiodifusión, sólo tenían reconocidos derechos patrimoniales respecto a autorizar o prohibir la reproducción directa o indirecta de sus fonogramas.

Asimismo, el artículo 16 del TOIEF dispone que las Partes podrán establecer en sus respectivas legislaciones las mismas limitaciones o excepciones previstas para la protección de los derechos de autor de las obras literarias y artísticas, respecto de la protección de los derechos de los artistas intérpretes o ejecutantes y los productores de fonogramas.

Por otro lado, este Acuerdo de manera diversa al TODA, definió la "puesta a disposición" como un derecho autónomo. En ese sentido, en la Declaración concertada relativa al artículo quince del Tratado, se determinó la imposibilidad de alcanzar una solución completa acerca del nivel de protección de los derechos de radiodifusión y comunicación al público que deben disfrutar los artistas, intérpretes o ejecutantes y los productores de fonogramas en el ámbito digital. Debido a la falta de consenso, los Estados Miembros decidieron dejar de lado dicha situación para resoluciones posteriores (OMPI, 2015: 3).

De esta forma, frente a la dificultad de prever las diversas modalidades de explotación de contenidos que surgen con el avance tecnológico, se ha buscado alcanzar determinado grado de regulación en el entorno digital, estableciendo marcos legales que regulen el flujo de información en línea como se hace en otros medios, como la radio y la televisión.

Al respecto, las propuestas para regular y monitorear el uso de internet fueron cobrando relevancia en el mundo con la presentación de iniciativas y acuerdos como la iniciativa de Ley contra la piratería en línea (Stop Online Piracy Act - SOPA), el proyecto de Ley para la prevención de amenazas en línea a la creatividad económica y el robo de la propiedad intelectual (Preventing Real Online Threats to Economic Creativity and Theft of Intellectual Property Act - PIPA), el Acuerdo Comercial Anti-Falsificación (Anti-Counterfeit Trade Agreement - ACTA), y otras regulaciones de carácter más bien regional de las que México sí es Parte, como el TIPAT y el T-MEC, entre otras, frente a las cuales los usuarios han sido los principales defensores de sus derechos de libre acceso a la información, libertad de

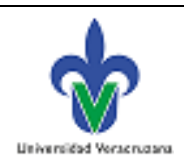

pp. $39-60$ 
ENFOQUES JURIDICOS

REVISTA MULTIDISCIPLINAR DEL CEDECS

ISSN 26832070

Número 04

julio-diciembre 2021
Artículo: "Los derechos de autor en el ámbito digital. Un necesario sistema de equilibrio de libertades en México"

José Joaquín Piña Mondragón

DOI: https://doi.org/10.25009/ej.v0i04.2570

expresión y acceso a internet, por la importancia que representa en la actualidad el compartir información y contenidos digitalmente, llevándolos a organizar auténticas acciones colectivas a través de las redes sociales para la defensa del ejercicio de libre acceso a la información que circula en internet.

En ese sentido, la Asamblea General de las Organización de las Naciones Unidas (ONU) declaró el acceso a Internet como un derecho humano, en los siguientes términos: "La única y cambiante naturaleza de Internet no sólo permite a los individuos ejercer su derecho de opinión y expresión, sino que también forma parte de sus derechos humanos y promueve el progreso de la sociedad en su conjunto". Al respecto, especificó que este derecho humano se viola de las siguientes formas: "a) con el bloqueo o filtros de contenidos; b) desconectando el acceso de manera premeditada o con ciberataques; y c) al no proteger el derecho de privacidad y la protección de datos en internet." (ONU, 2011: 14).

\section{c) Ley contra la piratería en línea (SOPA)}

La iniciativa de Ley H.R.3261 contra la piratería en línea, fue propuesta por Lamar S. Smith, presidente del Comité Judicial de la Cámara de Representantes al Congreso de los Estados Unidos de Norteamérica el 26 de octubre de 2011, con el propósito de disuadir la piratería de obras, persiguiendo las descargas ilegales de contenidos protegidos por copyright que circulan en internet. Lo anterior a través de diversas acciones como la restricción de las redes de publicidad en línea y los mecanismos de financiamiento de páginas web infractoras, e incluso autorizar a los proveedores de servicios de internet (PSI) para bloquear el acceso a sus páginas.

Dicho proyecto de ley consideraba como delito grave la transmisión no autorizada de contenidos protegidos con copyright, facultando al Departamento de Justicia de los Estados Unidos para solicitar órdenes judiciales e inhabilitar páginas web, incluso fuera de la jurisdicción de dicho país, al igual que obligar a los motores de búsqueda a eliminar los enlaces a sitios infractores.

Desde que se dio a conocer esta iniciativa surgieron opositores que la consideraron una amenaza directa a la libertad de expresión, de acceso a la información y de internet, al contemplar el bloqueo para acceder a un dominio completo por una sola infracción en una página web, situación que generó diversos reclamos que provocaron que sitios como eBay, Amazon, Wikipedia y Twitter suspendieran temporalmente sus páginas en protesta a esta iniciativa.

d) Ley para la prevención de amenazas en línea a la creatividad económica y el robo de la propiedad intelectual (PIPA)

Este proyecto de ley fue presentado el 12 de mayo de 2011 ante el Congreso estadounidense por parte del senador Patrick Leahy, con el propósito de brindar a las autoridades y a los titulares de copyright la posibilidad de restringir el acceso a usuarios de

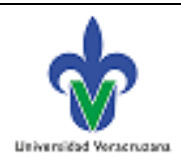

pp. $39-60$ 
ENFOQUES JURIDICOS

REVISTA MULTIDISCIPLINAR DEL CEDECS

ISSN 26832070

Número 04

julio-diciembre 2021
Artículo: "Los derechos de autor en el ámbito digital. Un necesario sistema de equilibrio de libertades en México"

José Joaquín Piña Mondragón

DOI: https://doi.org/10.25009/ej.v0i04.2570

páginas web que permitieran el almacenamiento o trasmisión de copias ilegales o tecnología que facilitara la evasión de las medidas tecnológicas de protección y la información sobre la gestión de derechos.

Los opositores a este proyecto argumentaron que la iniciativa atentaba contra la libertad de expresión e información de los usuarios de internet, al permitir implementar herramientas para eliminar o inhabilitar el acceso a la página web que almacenara contenido protegido por copyright sin autorización del titular (desde textos, imágenes letras de canciones, fotografías, etc.). En términos generales, las disposiciones de PIPA son similares a las de SOPA (Hernández, 2017: 6).

\section{e) Acuerdo Comercial Anti-Falsificación (ACTA)}

Este Acuerdo constituye un cuerpo normativo de carácter multilateral para la protección de los derechos de propiedad intelectual contra la falsificación de productos y la piratería de obras que circulan en internet, involucrando a los PSI en la disuasión de conductas como la descarga, transferencia e incluso la visualización de contenidos sin el pago de las regalías correspondientes.

En el año 2007, México se sumó a las negociaciones de este Tratado junto con los gobiernos de Australia, Canadá, Corea, Japón, Marruecos, Nueva Zelanda, Singapur, Suiza, Estados Unidos de Norteamérica y representantes de la Unión Europea, para posteriormente, ser firmado por el Ejecutivo en julio de 2012. Sin embargo, la Comisión Permanente del Congreso de la Unión rechazó la firma al considerar que dicho instrumento vulneraba derechos humanos como la libertad de expresión y acceso a la información contemplados en la Constitución.

\section{f) Tratado Integral y Progresista de Asociación Transpacífico (TIPAT)}

El también llamado TPP-11, debido a los 11 países de la región Asia-Pacífico que lo integran, constituye uno de los acuerdos comerciales de mayor relevancia que México ha suscrito en los últimos años. La importancia de este instrumento con relación a la protección de los derechos de autor y conexos en el ámbito digital, estriba en el reconocimiento de las llamadas Medidas Tecnológicas de Protección (MTP), previstas en el artículo 18.68 y la Información sobre la Gestión de Derechos (IGD) que establece el artículo 18.69, sendas disposiciones fueron incluidas en el Capítulo 18 de Propiedad Intelectual del Acuerdo, con el objeto de implementar tecnologías que permitan a los autores, artistas, intérpretes, ejecutantes o productores de fonogramas controlar de una manera más efectiva el acceso a sus obras y contenidos protegidos por derechos de autor y conexos, además de incluir información respecto a los términos y condiciones para su utilización en el entorno digital.

De igual forma, con el propósito de facilitar la observancia de los derechos de autor en internet y evitar alteraciones injustificadas al comercio en el entorno digital, el artículo 18.82 del Acuerdo, contempla incentivos legales a los PSI para cooperar con los titulares de

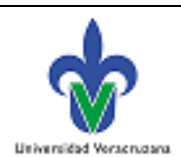

pp. $39-60$ 
ENFOQUES JURÍDICOS

REVISTA MULTIDISCIPLINAR DEL CEDECS

ISSN 26832070

Número 04

julio-diciembre 2021
Artículo: "Los derechos de autor en el ámbito digital. Un necesario sistema de equilibrio de libertades en México"

José Joaquín Piña Mondragón

DOI: https://doi.org/10.25009/ej.v0i04.2570

los derechos de autor en disuadir el almacenamiento y la transmisión no autorizadas de sus obras y contenidos, de manera que si formulan una reclamación legal satisfactoria de infracción al derecho de autor, puedan obtener la información que el PSI posea de manera expedita que les permita identificar al presunto infractor $y$, en su caso, iniciar las acciones legales correspondientes.

Al respecto, conviene señalar que algunos Estados han optado por establecer regulaciones para establecer puertos seguros a los PSI y dotar de herramientas a los titulares para proteger sus derechos de autor, en el caso de Estados Unidos, por ejemplo, con la Digital Millenium Act, y en la Unión Europea, con la Directiva 98/34/CE. Si bien estos modelos optaron por un mecanismo de protección más restrictivo, también puede generar abusos por parte de los PSI, afectando en determinado momento, el derecho a la privacidad, la libertad de expresión y el acceso a la información de los usuarios (Téllez, 2016: 236 y 237$)$.

\section{g) Tratado entre México, Estados Unidos y Canadá (T-MEC)}

El 1 de julio de 2020 entró en vigor el T-MEC, en sustitución del Tratado de Libre Comercio de América del Norte (TLCAN), con el propósito de establecer una zona de libre comercio de conformidad con los artículos XXIV del Acuerdo General sobre Aranceles Aduaneros y Comercio (GATT) y V del Acuerdo General sobre el Comercio de Servicios (GATS).

En materia de derechos de autor, este instrumento recoge varias de las disposiciones del TIPAT contenidas en el Capítulo 18, para plasmarlas en el Capítulo 20, con relación a la protección de los derechos de autor y conexos en el entorno digital. En ese sentido, el artículo 20.67 establece que cada Parte dispondrá los procedimientos administrativos y sanciones penales aplicables a quienes eludan las medidas tecnológicas de protección y la información sobre gestión de derechos que los titulares de derechos de autor y conexos empleen con relación a sus obras, interpretaciones y ejecuciones de fonogramas.

De igual forma, el artículo 20.89 involucra a los PSI a través de "incentivos" para cooperar con los titulares de derechos de autor en inhibir el almacenamiento y transmisión no autorizada de materiales protegidos por derechos de autor, incluyendo el retiro e inhabilitación del acceso a materiales alojados en sus sistemas o redes cuando tengan conocimiento cierto de la infracción al derecho de autor.

Al respecto, el T-MEC establece un mecanismo de notificación al presunto infractor cuyo material es removido o inhabilitado, teniendo éste la posibilidad de solicitar al PSI que restaure dicho material a través de una contra-notificación. En todo caso, los tres países Miembros asumieron el compromiso de incorporar en su legislación interna sanciones de carácter económico contra la persona que con el conocimiento de que realiza un señalamiento falso a través de una notificación o contra-notificación, lesione los intereses de cualquier parte interesada con motivo de que el PSI se haya apoyado en esa falsa

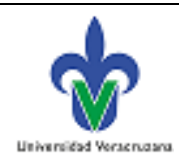

pp. $39-60$ 
ENFOQUES JURIDICOS

REVISTA MULTIDISCIPLINAR DEL CEDECS

ISSN 26832070

Número 04

julio-diciembre 2021
Artículo: "Los derechos de autor en el ámbito digital. Un necesario sistema de equilibrio de libertades en México"

José Joaquín Piña Mondragón

DOI: https://doi.org/10.25009/ej.v0i04.2570

notificación, para remover o inhabilitar el contenido presuntamente infractor o lesionar los derechos de los titulares.

Cabe destacar que estas disposiciones fueron recogidas con las recientes reformas a la LFDA en México, publicadas en el Diario Oficial de la Federación de fecha 1 de julio de 2020, mismas que por su trascendencia, analizamos a continuación.

\section{h) Ley Federal del Derecho de Autor (LFDA)}

Con la entrada en vigor del TIPAT, T-MEC y las recientes reformas a la LFDA, publicadas en el DOF del 1 de julio de 2020, se abre una nueva era en materia de regulación y protección del uso de obras y contenidos en el ámbito digital en México.

En ese sentido, la LFDA recogió las disposiciones contenidas en el Capítulo 18 del TIPAT y 20 del T-MEC, incorporando la remoción de obras y contenidos presuntamente infractores de derechos de autor y conexos, a través de un procedimiento que en la práctica se denomina como "notice and take down", que no es más que el descrito en el artículo 20.89 del T-MEC referente a la "notificación y contra-notificación".

Este procedimiento de aviso y contra-aviso establecido en el artículo 114 Octies de la LFDA, "incentiva" a los PSI para cooperar con los titulares en la disuasión del almacenamiento y la transmisión no autorizadas de materiales protegidos por derechos de autor y conexos. En concreto, el aviso que reciba el PSI por parte del titular para eliminar el acceso a contenidos habilitados o transmitidos sin su consentimiento debe al menos contener los siguientes requisitos:

a) Nombre del titular o representante legal y medio de contacto para recibir notificaciones.

b) Identificar el contenido de la infracción reclamada.

c) Manifestar el interés o derecho con respecto a los derechos de autor, y

d) Especificar los datos de la ubicación electrónica a la que se refiere la infracción reclamada (LFDA, artículo 114 Octies)

Al respecto, llama la atención que en ninguno de estos cuatro requisitos que establece la Ley con relación al aviso que presenta el titular o alguna persona autorizada para actuar en su representación, se hace referencia a la manera en que acreditará quien presente el aviso ante el PSI, la supuesta infracción que justifique la retirada del contenido, por lo que este último simplemente deberá remover, retirar, eliminar o inhabilitar el acceso al material supuestamente infractor, incluso sin contar con elementos de prueba.

Por su parte, el usuario cuyo contenido fue eliminado, tiene la posibilidad a través de un contra-aviso de solicitar al PSI la restauración del contenido, debiendo demostrar para ello la titularidad o autorización con que cuenta para ese uso específico del material removido o, en su caso, justificar su uso conforme a las limitaciones o excepciones a los derechos que la misma Ley prevé.

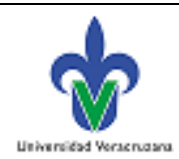

pp. $39-60$ 
ENFOQUES JURIDICOS

REVISTA MULTIDISCIPLINAR DEL CEDECS

ISSN 26832070

Número 04

julio-diciembre 2021
Artículo: "Los derechos de autor en el ámbito digital. Un necesario sistema de equilibrio de libertades en México"

José Joaquín Piña Mondragón

DOI: https://doi.org/10.25009/ej.v0i04.2570

Por otro lado, la reforma tampoco especifica la manera en que el titular o su representante que presenten el aviso al PSI acreditará su personalidad, por lo que, en la práctica, pudieran darse supuestos en que cualquier persona suplantando la identidad del titular o representante, presenten el aviso al PSI sin previamente comprobar la titularidad o representación respecto del contenido removido o inhabilitado.

Con relación a este procedimiento de notificación y contra-notificación, no pasa desapercibido que el artículo 232 quinquies prevé la imposición de multas para quien realice una falsa declaración de aviso o contra-aviso, que afecte a cualquiera de las partes interesadas debido a que el PSI se basó en la misma, ya sea para remover el contenido, o bien, para rehabilitarlo a través del contra-aviso.

Otro de los aspectos relevantes sobre los que versa la reforma a la LFDA, respecto a la protección de las obras en el ámbito digital, es la relativa a la incorporación de las Medidas tecnológicas de protección o candados digitales que permitan controlar de manera más efectiva el acceso a una obra o contenido. En ese sentido, la ruptura de estos candados supone una conducta ilegal y se encuentra sujeta a la imposición de multas.

De igual forma, a través de la información sobre la gestión de derechos o "huella dactilar" de las obras, será posible identificar plenamente a la obra, su autor y al titular de cualquier derecho sobre los mismos, así como obtener información respecto de los términos y condiciones de utilización de las obras.

Finalmente, vale la pena mencionar que el 3 de agosto de 2020 la Comisión Nacional de Derechos Humanos (CNDH), presentó acción de inconstitucionalidad ante la Suprema Corte de Justicia de la Nación (SCJN), con relación a algunos artículos de la reforma a la LFDA, por considerar que violan derechos fundamentales como la seguridad jurídica, libertad de expresión y debido proceso. De manera particular, con relación al artículo 114 Octies, fracción II, inciso a), por considerar que vulnera la seguridad jurídica al no precisar los alcances de remover, retirar, eliminar e inhabilitar aquellos contenidos presuntamente infractores de los derechos de autor y conexos ni proporcionar los lineamientos mínimos para que los destinatarios de la norma, conozcan los supuestos en que podrá operar alguna de éstas, pudiendo generar que los PSI de manera discrecional y arbitraria lleven a cabo estas acciones en perjuicio de los usuarios de internet.

En efecto, la CNDH considera que las reformas a la LFDA que derivaron del proceso de armonización legislativa que tuvo lugar con la entrada en vigor del T-MEC, podrían vulnerar derechos fundamentales con relación al mecanismo de aviso y retirada de información alojada en los sistemas o redes de los PSI que se considere presuntamente infractora de derechos de autor, lo cual puede generar censura de opiniones y la libre circulación de las ideas a través de internet debido a una deficiente regulación en la que incluso no se refiere la manera en que la persona que presenta el aviso acreditará el interés jurídico o la titularidad de los derechos de autor presuntamente infringidos. Aunado a lo anterior, no se

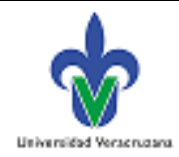

pp. $39-60$ 
ENFOQUES JURIDICOS

REVISTA MULTIDISCIPLINAR DEL CEDECS

ISSN 26832070

Número 04

julio-diciembre 2021
Artículo: "Los derechos de autor en el ámbito digital. Un necesario sistema de equilibrio de libertades en México"

José Joaquín Piña Mondragón

DOI: https://doi.org/10.25009/ej.v0i04.2570

establece con precisión aquellos elementos que el PSI valorará y tomará en cuenta para determinar si efectivamente se cometió la infracción denunciada.

En ese sentido, la Comisión advierte que se genera incertidumbre con relación a la forma en que la persona que formula un aviso respecto al contenido presuntamente infractor demuestre tener interés jurídico o bien ser titular del derecho presuntamente infringido, configurando una especie de sistema aparentemente probatorio en el cual existe un total desequilibrio entre las partes.

\section{Derechos de autor y TIC}

La autorización de la copia privada y las limitaciones y excepciones al derecho de autor reconocidas por aquellos países que adoptaron el Convenio de Berna o la limitación genérica del fair use del derecho anglosajón, que permiten un uso limitado de las obras protegidas por derechos de autor sin necesidad de contar con la autorización del titular y de manera gratuita, para uso académico e informativo, por ejemplo, constituyó el paradigma que durante varios años permitió equilibrar las relaciones entre los titulares de los derechos de autor y conexos y los usuarios de las obras. Sin embargo, en el ámbito digital se presentaron nuevamente diferencias por la facilidad que representa para los usuarios acceder a obras y contenidos de manera gratuita a través de internet (García, 2019: 63).

Ante esta situación, se ha buscado establecer un sistema de equilibrio frente al aumento exponencial que ha tenido la comunicación, fijación, reproducción, distribución y comunicación pública de las obras y contenidos en internet sin remuneración alguna para sus titulares. No obstante, también es una realidad que el entorno digital ofrece a los autores nuevas formas de explotación y comercialización de sus obras que les representan fuentes adicionales de retribución.

En efecto, con los avances tecnológicos los derechos de autor adquieren nuevas formas de tratamiento, debido a los medios en los que se comercializan, reproducen y utilizan las obras y los soportes en que se fijan. Lo anterior genera la necesidad de equilibrar el ejercicio de esos derechos frente al desarrollo tecnológico que plantea la actual sociedad del conocimiento. La importancia de proteger los derechos de autor en el ámbito digital deviene en muchas ocasiones de intereses de carácter económico, incluso las iniciativas de protección, por lo general, son impulsadas por empresas que gestionan los derechos patrimoniales de los autores.

Al respecto, considerando la necesaria ponderación entre los derechos de autor y las libertades de los usuarios que acceden a las obras y contenidos en el entorno digital, resulta importante enfatizar las principales consecuencias que la emergencia de las TIC ha provocado en el esquema tradicional de protección de estos derechos:

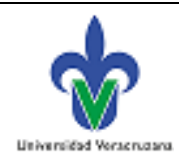

pp. $39-60$ 
ENFOQUES JURIDICOS

REVISTA MULTIDISCIPLINAR DEL CEDECS

ISSN 26832070

Número 04

julio-diciembre 2021
Artículo: "Los derechos de autor en el ámbito digital. Un necesario sistema de equilibrio de libertades en México"

José Joaquín Piña Mondragón

DOI: https://doi.org/10.25009/ej.v0i04.2570

- Implica nuevos medios o soportes a través de los cuales se expresan las obras intelectuales. En la actualidad, es común que un libro se presente no sólo en formato impreso, sino también en formato electrónico descargable.

- Posibilita nuevos usos respecto de una misma obra intelectual. Por ejemplo, un libro puede ser escaneado, leído desde la pantalla de un dispositivo electrónico, enviado por correo electrónico, almacenado en la nube, impreso, etc.

- Facilita la copia indiscriminada de obras intelectuales de la misma calidad, o incluso superior.

- Permite una comunicación e intercambio sin fronteras de obras protegidas, lo cual favorece la transmisión y comercio de estas.

- Ha generado el inicio de un fenómeno digitalizador de las obras intelectuales, facilitando su almacenamiento y propagación.

En la actualidad, es posible acceder a diversas obras a través de internet sin prácticamente restricción alguna, pudiendo reproducirse ilimitadamente y sin menoscabo de su calidad, por lo que el control y vigilancia por parte de los titulares y de las autoridades se vuelve cada vez más difícil. De esta forma, la puesta a disposición de las obras y contenidos a través de internet dificulta la protección de los derechos de autor, ya que nuevas tecnologías facilitan a los usuarios la elusión de medidas tecnológicas de protección y la información sobre gestión de derechos, permitiendo la propagación y acceso a las obras de los creadores, brindando la opción de adaptar, almacenar, copiar, transformar y comunicar las obras de manera automática y gratuita.

Por lo anterior, con el objeto de restringir el uso no autorizado, se han creado disposiciones para la protección de los derechos de autor y conexos en el ámbito digital, que tienen como finalidad la protección de los derechos de autor en el mundo y la implementación de medidas tecnológicas con ese propósito. Ejemplos de dicha protección serían una contraseña de acceso, una marca de agua o un dispositivo informático que impide al usuario hacer una copia digital de la obra. Sin embargo, para que las medidas tecnológicas puedan proteger eficazmente las obras en el entorno digital es necesario que se sancione su elusión, pues de otro modo la obra puede ser despojada de su protección al momento de eludir dichas tecnologías (Bercovitz, 2006: 316).

\section{La protección de los derechos de autor frente a los derechos y las libertades fundamentales de los usuarios en el ámbito digital}

Un régimen eficaz en materia de protección de los derechos de autor y conexos en el entorno digital debe procurar, por un lado, un equilibrio entre la creatividad y promoción de obras y contenidos originales, y por otro, proteger las libertades fundamentales de los usuarios en internet, como las de información, expresión y promoción de la cultura y

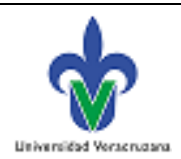

pp. $39-60$ 
ENFOQUES JURIDICOS

REVISTA MULTIDISCIPLINAR DEL CEDECS

ISSN 26832070

Número 04

julio-diciembre 2021
Artículo: "Los derechos de autor en el ámbito digital. Un necesario sistema de equilibrio de libertades en México"

José Joaquín Piña Mondragón

DOI: https://doi.org/10.25009/ej.v0i04.2570

educación. Para el logro de estos objetivos, dicho régimen ha de brindar un sólido respaldo a los derechos de autor y contenidos digitales, aunque sujeto a un conjunto de límites a los mismos.

Los derechos patrimoniales o de explotación, como la reproducción, distribución, comunicación pública y transformación, bajo circunstancias particulares expresamente señaladas en instrumentos internacionales como el Convenio de Berna, han sido limitados con el propósito de encontrar un equilibrio entre los autores y/o titulares de derechos, que buscan la más amplia protección de sus obras, y los usuarios que desean acceder igualmente a ellas con mínimas restricciones.

En ese contexto, el régimen de limitaciones y excepciones al derecho de autor ha constituido uno de los mecanismos más importantes en la búsqueda de ese equilibrio entre los derechos de los autores y creadores de contenidos digitales y el interés público de los usuarios en general. No se trata simple y llanamente de excepciones a los derechos, pues en la práctica implican auténticas libertades fundamentales e intereses superiores de la sociedad, pues sin excepciones y limitaciones, el sistema de derechos de autor no podría lograr su cometido fundamental, que es estimular la creación de obras originales para beneficio de la humanidad.

Vale la pena resaltar que, en contraposición a los derechos de explotación, los derechos morales (divulgación, paternidad, integridad y retirada de la circulación) son personalísimos, y de acuerdo con los artículos 18 y 19 de la LFDA, se consideran unidos al autor, siendo inalienables, imprescriptibles, irrenunciables e inembargables; por lo que no están sujetos a limitación alguna y las excepciones aplicables a los derechos patrimoniales sobre las obras no los afectan de forma alguna (De la Parra, 2014: 56).

En ese sentido, aspectos como la digitalización de contenidos, las nuevas formas de circulación y difusión de las obras y el aumento de la velocidad de transmisión y tráfico a través de internet, han impactado de manera importante la protección de los derechos de autor y conexos, orillando a un replanteamiento en torno a los mismos y a endurecer y buscar nuevas formas para su protección, como es el caso de las medidas de carácter tecnológico y la información sobre la gestión de derechos (Barrenechea, 2017: 56).

El conjunto de excepciones previstas en la Ley se considera de interés público, pues tienen por objeto lograr un equilibrio entre los intereses de los autores y de los usuarios y público en general, salvaguardando sus derechos fundamentales, como la libertad de expresión e información y el acceso a la cultura y al conocimiento. No obstante, dichas excepciones están sujetas a numerus clausus y su regulación debe interpretarse en forma restrictiva, puesto que, bajo ciertas condiciones, implican una disminución a la protección de los derechos patrimoniales, dado que pueden ejercerse sin requerir el consentimiento previo y expreso de sus autores y/o titulares. Las limitaciones pueden ser de dos tipos; por

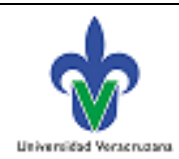

pp. $39-60$ 
ENFOQUES JURÍDICOS

REVISTA MULTIDISCIPLINAR DEL CEDEGS

ISSN 26832070

Número 04

julio-diciembre 2021
Artículo: "Los derechos de autor en el ámbito digital. Un necesario sistema de equilibrio de libertades en México"

José Joaquín Piña Mondragón

DOI: https://doi.org/10.25009/ej.v0i04.2570

un lado, las utilizaciones libres y gratuitas, como el derecho de cita; y por otro, aquellas que están sujetas a remuneración, denominadas licencias no voluntarias (LIPSZYC, 1993: 20).

En ese contexto, se ha buscado la armonización de la legislación en la materia, estableciendo, por un lado, estándares de protección a los derechos de autor, y por otro, se ha regulado el ámbito de las limitaciones y excepciones con distinto alcance y nivel. Particularmente, el Convenio de Berna para la Protección de Obras Artísticas y Literarias, establece en los artículos 10 y 10 bis diversos supuestos en los que es posible la libre utilización de obras sin la autorización del titular y de manera gratuita, como el derecho de cita, la posibilidad de utilizar obras artísticas o literarias con propósitos de enseñanza, la reproducción y difusión por la prensa, las noticias, etcétera.

Asimismo, desde el Acta de Estocolmo del 14 de julio de 1967 y que se mantuvo en el Acta de París del 24 de julio de 1971, se estableció en el artículo $9^{\circ}$, párrafo 2 , una disposición específica que se mantiene vigente hasta la actualidad, en torno a las excepciones aplicables al derecho de reproducción, denominada "regla de los 3 pasos", conforme a la cual los Miembros de la Unión de Berna están facultados para establecer en sus respectivas legislaciones, excepciones respecto al derecho de reproducción, si cumplen con los siguientes requisitos:

a) Solo para casos especiales (carácter excepcional);

b) No atente a la explotación normal de la obra; y

c) No cause un perjuicio injustificado a los intereses legítimos del autor.

Este mecanismo fue adoptado no solamente respecto del derecho de reproducción, sino que fue extendido al resto de los derechos patrimoniales, en el ADPIC de la OMC, en el TODA y, en lo referente a los derechos conexos de los artistas, intérpretes y ejecutantes y los productores de fonogramas en el TOIEF, que abordaron la denominada agenda digital y reconocieron que las excepciones aplicables en el entorno analógico tienen plena vigencia en el digital.

En México, en el artículo 148 de la LFDA, se establecen los supuestos específicos respecto la limitación de los derechos patrimoniales, en los siguientes términos: "Las obras literarias y artísticas ya divulgadas podrán utilizarse, siempre que no se afecte la explotación normal de la obra, sin autorización del titular del derecho patrimonial y sin remuneración, citando invariablemente la fuente y sin alterar la obra (...)", haciendo referencia a la cita parcial de textos; la reproducción de artículos, fotografías, ilustraciones y comentarios publicados y difundidos siempre que no medie prohibición expresa por parte del titular; la reproducción parcial de la obra para la crítica e investigación científica o literaria; la reproducción por única ocasión y en ejemplar único de una obra para uso personal, privado y sin fines de lucro; la reproducción para constancia en un procedimiento judicial o administrativo; la reproducción, comunicación y distribución a través de imágenes de las obras para hacerlas

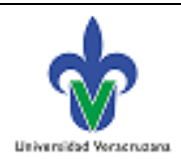

pp. $39-60$ 
ENFOQUES JURIDICOS

REVISTA MULTIDISCIPLINAR DEL CEDECS

ISSN 26832070

Número 04

julio-diciembre 2021
Artículo: "Los derechos de autor en el ámbito digital. Un necesario sistema de equilibrio de libertades en México"

José Joaquín Piña Mondragón

DOI: https://doi.org/10.25009/ej.v0i04.2570

visibles desde lugares públicos; y la publicación de obras artísticas y literarias sin fines de lucro para personas con discapacidad.

De esta manera, el TODA recogió las limitaciones y excepciones a los derechos patrimoniales reconocidas en el Convenio de Berna, sujetándolas al requisito del cumplimiento de la regla de los tres pasos. De igual forma, mediante una Declaración Concertada relativa al artículo 10 del TODA, la cual tiene un equivalente en el TOIEF para los derechos de los intérpretes o ejecutantes y los productores de fonogramas, se estableció el supuesto en torno a la explotación de obras en el entorno digital, al disponer que las Partes contratantes "podrán aplicar y ampliar debidamente las limitaciones y excepciones al entorno digital", inclusive para "establecer nuevas excepciones y limitaciones que resulten adecuadas al entorno de red digital." (OMPI, 2005: 10).

Como señala Fícsor,

la aplicabilidad y extensión de las limitaciones y excepciones "existentes» deben ser revisadas cuando ellas son "trasladadas" al entorno digital. El resultado de tal revisión puede ser que el alcance de aplicación de las limitaciones y excepciones sea reducido (o, tal vez justamente lo opuesto, como lo subraya la declaración concertada, en algunos aspectos especiales ampliados (Fícsor, 2002: 13).

En efecto, el ejercicio de los derechos de acceso a la información, la libertad de expresión y de prensa y el respeto a la vida privada de los usuarios, también implica limitaciones al derecho de autor en el ámbito digital, tomando en cuenta que cada vez son más las obras y contenidos disponibles a través de internet esta situación adquiere relevancia. Por ello, es importante que los estudiantes, investigadores y la comunidad científica, en principio tengan acceso y se beneficien de las mismas oportunidades respecto el acceso a las obras tanto en el ámbito digital como en el analógico. Lo anterior justifica el reconocimiento de las excepciones que benefician tanto a las bibliotecas como a la comunidad científica y educativa (Dusollier, 2001: 14).

En la actualidad, asistimos a un incremento notable en la cantidad de información y contenidos que se producen en el ámbito digital; sin embargo, el acceso de los usuarios a los contenidos, la información y la cultura, no puede concebirse en un ambiente de inobservancia de los derechos de los titulares, pues si bien el acceso a la información y la libertad de expresión a través de internet representa uno de los pilares de la sociedad del conocimiento, las libertades y derechos de los usuarios deben enmarcarse en el pago de una retribución justa para los creadores por el uso que se hace de sus obras (Farré, 2008: 69).

Con base en lo anterior, resulta claro que la protección de obras y contenidos en el contexto de la sociedad del conocimiento no es una cuestión pacífica, pues se encuentra inmersa en un cambio de paradigma tecnológico que afecta de manera significativa la comunicación pública de los contenidos en el entorno digital, en donde terminan

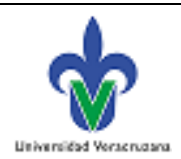

pp. $39-60$ 
ENFOQUES JURIDICOS

REVISTA MULTIDISCIPLINAR DEL CEDECS

ISSN 26832070

Número 04

julio-diciembre 2021
Artículo: "Los derechos de autor en el ámbito digital. Un necesario sistema de equilibrio de libertades en México"

José Joaquín Piña Mondragón

DOI: https://doi.org/10.25009/ej.v0i04.2570

confluyendo otros derechos como la libertad de expresión, información y el derecho de acceso a la cultura y educación a través de internet.

En ese sentido, el reto en el corto y mediano plazo consiste en encontrar una fórmula que garantice la protección de los derechos de los autores respecto de sus obras, sin lesionar los derechos y libertades fundamentales de los usuarios. Posiblemente, la respuesta requerirá concebir nuevas fórmulas para la remuneración de las obras y contenidos digitales, ya que las TIC han alterado sustancialmente las tradicionales.

\section{Conclusiones}

La protección de los derechos de autor en el ámbito digital presenta dos perspectivas, por un lado, un reclamo legítimo y generalizado de los autores y creadores de contenidos, quienes consideran que en muchas ocasiones la retribución por el acceso y comunicación pública de sus obras no llega a sus manos; y por otro, los usuarios que en ejercicio de sus libertades de acceso a la información, educación y difusión de la cultura, acceden a las obras y contenidos a través de internet, no deben ser considerados por ese hecho, como potenciales infractores de los derechos de autor, sino como quienes financian y estimulan la creación y distribución de obras en el entorno digital.

Sin lugar a duda, la protección de los derechos de autor en el entorno digital exige un diálogo multilateral entre los países, sin perder de vista el equilibrio que necesariamente debe existir entre los intereses de los titulares y los derechos y libertades de los usuarios, con el propósito de establecer un sistema de protección más amplio y eficaz, que a la vez tome en cuenta que las TIC son fundamentales para el desarrollo de la industria de contenidos. Al respecto, la difusión de la información, la cultura e incluso la transferencia de tecnología en la sociedad del conocimiento, tampoco pueden dejar de lado el hecho de que los derechos de propiedad intelectual giran en torno al derecho de acceso a la información, la educación, la libertad de expresión y de internet.

Por tanto, la protección de los derechos de autor debe considerarse como un fin legítimo que puede dar lugar a la imposición de limitaciones al derecho a la libertad de expresión, siempre y cuando, no se convierta en una restricción o censura previa de tales contenidos y, por lo mismo, en una restricción injustificada o desproporcionada de dicho derecho humano.

Por esas razones y con la finalidad de que la limitación al derecho a la información sea legítima, necesaria y proporcional, cuando exista posible violación a los derechos de autor, fue que recientemente se reformó la LFDA, estableciendo los procedimientos con antelación descritos, en donde se da la posibilidad al usuario de internet cuyo contenido ha sido retirado, bloqueado o eliminado a ejercer de manera efectiva su derecho de respuesta o rectificación mediante un "contra-aviso", en el que demuestre la titularidad o autorización del uso específico del contenido o bien, justificar su uso de acuerdo con las limitaciones o excepciones a los derechos de autor.

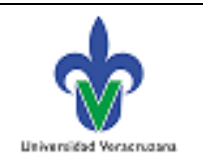

pp. $39-60$ 
ENFOQUES JURÍDICOS

REVISTA MULTIDISCIPLINAR DEL CEDEGS

ISSN 26832070

Número 04

julio-diciembre 2021
Artículo: "Los derechos de autor en el ámbito digital. Un necesario sistema de equilibrio de libertades en México"

José Joaquín Piña Mondragón

DOI: https://doi.org/10.25009/ej.v0i04.2570

Con base en lo anterior, consideramos que la legislación en materia de derechos de autor debe consolidarse con las recientes reformas como un instrumento que no sólo garantice la protección efectiva de los autores y los incentive a seguir creando obras originales, sino que además permita a los usuarios acceder a contenidos de interés cultural y educativo que contribuya con el fin de construir una sociedad del conocimiento más abierta y equilibrada. No obstante, para que esta meta sea posible es necesario consolidar un sistema que estimule el pago de una retribución justa para los creadores, autores y titulares de los derechos autorales y garantice el acceso a la información, educación y cultura a los usuarios. Aspiramos al desarrollo de una sociedad en la que la tecnología se consolide no como un obstáculo, sino como un medio para la construcción del conocimiento. 
ENFOQUES JURIDICOS

REVISTA MULTIDISCIPLINAR DEL CEDEGS

ISSN 26832070

Número 04

julio-diciembre 2021
Artículo: "Los derechos de autor en el ámbito digital. Un necesario sistema de equilibrio de libertades en México"

José Joaquín Piña Mondragón

DOI: https://doi.org/10.25009/ej.v0i04.2570

\section{Fuentes de Consulta}

Barrenechea, A. (2017). Régimen de excepciones y limitaciones al Derecho de los Autores. Perú. Revista Derecho \& Sociedad Pontificia Universidad Católica del Perú. No. 49. Recuperado de http://revistas.pucp.edu.pe/index.php/derechoysociedad/article/view/19877

Barrera, M. (2005). Derechos de Autor y Libertad de Expresión. Ecuador. Chasqui Revista Latinoamericana de Comunicación Centro Internacional de Estudios Superiores de Comunicación para América Latina de Quito. No. 90. Recuperado de https://revistachasqui.org/index.php/chasqui/article/view/238

Bercovitz, R. (Coord.). (2006). Manual de Propiedad Intelectual. Valencia, España. Tirant Lo Blanch.

De la Parra, E. (2014). Introducción al derecho intelectual. México. Porrúa/UNAM.

Dusollier, S. (2001). Derecho de autor y acceso a la información en el ámbito digital. Chile. Revista Derecho y tecnologías del Centro de Estudios en Derecho Informático de la Facultad de Derecho de la Universidad de Chile. Recuperado de http://www.crid.be/pdf/public/4133.pdf

Farré, P. (2008). Los Derechos de Autor en Internet. España. Anuario Facultad de Derecho. Universidad de Alcalá I.

Fícsor, M. (2002). La protección del derecho de autor y los derechos en el entorno digital. El Tratado de la OMPI sobre Derecho de Autor (WCT) y el Tratado de la OMPI sobre Interpretación o Ejecución y Fonogramas (WPPT). Limitaciones o excepciones en el ámbito digital. Panamá. Documento OMPI/DA/PAN/02/PIII.2, IV Congreso Iberoamericano sobre Derecho de Autor y Derechos Conexos (La Propiedad Intelectual, un canal para el desarrollo).

García, J. (2019). Derechos de autor en Internet. México. UNAM/Coordinación General de Estudios de Posgrado.

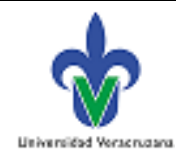

pp. $39-60$ 
ENFOQUES JURIDICOS

REVISTA MULTIDISCIPLINAR DEL CEDEGS

ISSN 26832070

Número 04

julio-diciembre 2021
Artículo: "Los derechos de autor en el ámbito digital. Un necesario sistema de equilibrio de libertades en México"

José Joaquín Piña Mondragón

DOI: https://doi.org/10.25009/ej.v0i04.2570

Guerra, J. y Hernández, J. (Coords.). (2021). La información después de internet. Repensando las libertades, amenazas y derechos. México. Colección Sistemas Bibliotecarios de Información y Sociedad. Instituto de Investigaciones Bibliotecológicas y de la Información/UNAM.

Hernández, J. (2017). Amenazas a la infodiversidad en Internet: censura y regulaciones contra el libre acceso a la información. En Morales, E. (Coord.). Regulaciones que impactan a la infodiversidad y el acceso abierto a la información en la sociedad global y multicultural. México. UNAM. Recuperado de http://iibi.unam.mx/publicaciones/278/regulaciones\%20impactan\%20infodiversidi n\%20Amenazas\%20en\%20Internet\%20Jonathan\%20Hernandez\%20Perez.html

Lasprilla B. (et. al.). (2012). S.O.P.A. Fenómeno social de actualidad. Colombia. Revista del Programa de Psicología de la Universidad del Norte, vol. 29, No. 1, enero-abril. Barranquilla. Recuperado de https://www.redalyc.org/pdf/213/21323171001.pdf

Lipszyc, D. (1993). Derecho de autor y derechos conexos. Buenos Aires, Argentina. UNESCO/CERLALC/Zavalia.

Mata y Martín, R. (et. al.). (2011). La propiedad intelectual en la era digital. Limites e infracciones a los derechos de autor en internet. España. La Ley/Grupo Wolters Kluwer.

Morales, E. (et. al.). (2006). La Propiedad Intelectual y los Derechos de Autor: Iniciativas de Organismos y Asociaciones Internacionales. Buenos Aires, Argentina. Revista Infodiversidad. No. 10. Sociedad de Investigaciones Bibliotecológicas.

Rubí, N. (2007). Las nuevas tecnologías en el derecho de autor y su evolución en Colombia. Bogotá, Colombia. Revista Prolegómenos. Derechos y Valores. Vol. X. No. 19, enerojunio. Universidad Militar Nueva Granada.

Solana, D. (2006). El Derecho de la Propiedad Intelectual y las Nuevas Tecnologías: Evolución y retos. España. Revista de Contratación Electrónica. No. 67. Dykinson. Recuperado de http://www.interiuris.com/blog/wp-content/uploads/Rce-67-4.pdf

Soler, F. (2018). Infracciones a los derechos de autor en México con relación al entorno digital. España. Ius Et Sientia. Revista electrónica de Derecho y Ciencia. Vol. 4. No. 1. Universidad de Sevilla.

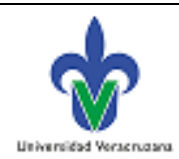

pp. $39-60$ 
ENFOQUES JURIDICOS

REVISTA MULTIDISCIPLINAR DEL CEDEGS

ISSN 26832070

Número 04

julio-diciembre 2021
Artículo: "Los derechos de autor en el ámbito digital. Un necesario sistema de equilibrio de libertades en México"

José Joaquín Piña Mondragón

DOI: https://doi.org/10.25009/ej.v0i04.2570

Téllez, E. (Coord.). (2016). Derecho y TIC. Vertientes actuales. México. Instituto de Investigaciones Jurídicas de la UNAM/INFOTEC.

\section{Legisgrafía}

Comisión Nacional de los Derechos Humanos. (agosto de 2020). Acción de Inconstitucionalidad en contra de los artículos 114 Quáter, 114 Quinquies, 114 Octies, fracciones II, inciso a) -salvo su numeral 2- y b), y III, 232 Bis y 232 Ter de la Ley Federal del Derecho de autor.

Diario Oficial de la Federación. (20 de febrero de 2018). Capítulo 18. Propiedad Intelectual. Tratado Integral y Progresista de Asociación Transpacífico. Recuperado de https://www.gob.mx/cms/uploads/attachment/file/86486/18._Propiedad_Intelect ual.pdf.

Diario Oficial de la Federación. (29 de junio de 2020). Capítulo 20. Derechos de Propiedad Intelectual. Tratado entre México, Estados Unidos y Canadá (T-MEC). Recuperado de https://www.gob.mx/cms/uploads/attachment/file/465802/20ESPDerechosdePro ESPDerechosdeProp.pdf.

Ley Federal del Derecho de Autor. México. Recuperado de http://www.diputados.gob.mx/LeyesBiblio/pdf/122_010720.pdf.

Organización Mundial de Comercio. (abril de 1994). Acuerdo sobre los aspectos de los derechos de propiedad intelectual relacionados con el comercio. Anexo 1C. Ginebra, Suiza. Recuperado de https://www.wto.org/spanish/docs_s/legal_s/27-trips.pdf.

Organización Mundial de la Propiedad Intelectual. (Enmendado el 28 de septiembre de 1979). Convenio de Berna para la Protección de las Obras Literarias y Artísticas. Ginebra, Suiza. Recuperado de https://wipolex.wipo.int/es/text/283694.

Organización Mundial de la Propiedad Intelectual. (7 al 11 de noviembre de 2005). XI Curso Académico Regional OMPI/SGAE sobre Derecho de Autor y Derechos Conexos para países de América Latina: El derecho de autor y los derechos conexos en el entorno digital. Asunción, Paraguay.

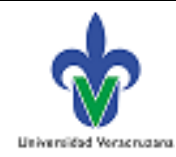

pp. $39-60$ 
ENFOQUES JURIDICOS

REVISTA MULTIDISCIPLINAR DEL CEDEGS

ISSN 26832070

Número 04

julio-diciembre 2021
Artículo: "Los derechos de autor en el ámbito digital. Un necesario sistema de equilibrio de libertades en México"

José Joaquín Piña Mondragón

DOI: https://doi.org/10.25009/ej.v0i04.2570

Organización Mundial de la Propiedad Intelectual. (2015). Propuesta de análisis de los derechos de autor en el entorno digital presentada por el Grupo de Países de América Latina y el Caribe. Ginebra, Suiza. Comité Permanente de Derecho de Autor y Derechos Conexos. XXXI Sesión.

Organización de las Naciones Unidas. (junio de 2011). Promoción y protección del derecho a la libertad de opinión y de expresión. Ginebra, Suiza. Comunicado de Prensa. Asamblea General. Sexagésimo sexto período de sesiones.

Organización Mundial de la Propiedad Intelectual. (1996). Tratado de la OMPI sobre Derecho de Autor. Ginebra, Suiza. Recuperado de https://www.wipo.int/edocs/lexdocs/treaties/es/wct/trt_wct_001es.pdf.

Organización Mundial de la Propiedad Intelectual. (1996). Tratado de la OMPI sobre Interpretación o Ejecución y Fonogramas. Ginebra, Suiza. Recuperado de https://www.wipo.int/edocs/lexdocs/treaties/es/wppt/trt_wppt_001es.pdf. 